\title{
Accretion of radiation and rotating Primordial black holes
}

\author{
Swapna Mahapatra and Bibekananda Nayak \\ Department of Physics, Utkal University, Bhubaneswar 751004, India \\ swapna@iopb.res.in, bibeka@iopb.res.in
}

\begin{abstract}
We consider rotating Primordial black holes (PBHs) and study the effect of accretion of radiation in the radiation dominated era. The central part of our analysis deals with the role of the angular momentum parameter on the evolution of PBHs. We find that both the accretion rate and evaporation rate decrease with increase in angular momentum parameter, but the rate of evaporation decreases more rapidly than the rate of accretion. This shows that the evaporation time of PBHs get prolonged with increase in angular momentum parameter. We also note that the lifetime of rotating $\mathrm{PBH}$ increase with increase in accretion efficiency of the radiation as in the case of nonrotating PBHs.
\end{abstract}




\section{Introduction}

Primordial black holes (PBHs) are supposed to be formed during the early expansion of the universe. These black holes may have been produced due to density fluctuation in the early universe with extremely high temperature and pressure. The mass of the PBHs can cover a wide range. There are different theories regarding the formation of PBHs such as, initial inhomogeneities [1, 2], inflation [3, 4], phase transitions [5] bubble collisions [6, 7], decay of cosmic loops [8] etc. The formation of PBH can also play a very important role in understanding the cosmological inflation. According to the work of Stephen Hawking, black holes emit thermal radiation due to quantum effects near the event horizon [9]. As a result of Hawking radiation, the black holes can lose mass and evaporate. Smaller mass black holes are expected to evaporate quickly. The PBHs with a longer life time can act as seeds for structure formation [10]. PBHs with mass greater than $10^{15} \mathrm{~g}$ do not evaporate completely through Hawking radiation and the abundance of such black holes can be considered as suitable dark matter candiadate [11].

In the context of standard cosmology, early work on the study of the effect of accretion of radiation on PBHs has led to several speculations regarding the possibility of increasing the mass of a PBH [1, 12]. Cosmological consequences of evaporation of PBHs in different eras have been studied quite well [13, 14] (see [15] for new cosmological constraints on PBHs). It has been realized during last couple of years that the effect of accretion in the radiation dominated era can result in longlived PBHs in braneworld scenario [16], in Brans-Dicke theory [17, 18, 19] as well as in standard cosmology [20]. The impact of accretion of phantom energy and vacuum energy on the evolution of PBH has also been discussed in refs [21, 22].

In this paper, we study the evolution of rotating PBHs in the context of standard cosmology by including the effect of accretion of radiation. We obtain the dependence of the evaporation time on the accretion efficiency and the angular momentum parameter. It is found that the evaporation time of the rotating $\mathrm{PBHs}$ gets prolonged both due to the increase in angular momentum parameter and accretion efficiency.

\section{Rotating PBHs and accretion of radiation}

In Einstein-Maxwell theory, the most general black hole solutions with mass, nonzero charge and angular momentum are described by the Kerr-Newman space time. Here, we consider uncharged, rotating PBHs in the context of spatially flat FRW Universe. We assume that the universe is filled with a perfect fluid described with the equation

of state $p=\gamma \rho$ (where $\gamma=\frac{1}{3}$ for the radiation dominated era and $\gamma=0$ for the matter 
dominated era). The Einstein equation is given by,

$$
\left(\frac{\dot{a}}{a}\right)^{2}=\frac{8 \pi G}{3} \rho
$$

The energy-momentum conservation equation is given by,

$$
\dot{\rho}+\left(\frac{3 \dot{a}}{a}\right)(1+\gamma) \rho=0
$$

where $a(t)$ is the scale factor. From the above equations, one finds that the scale factor $a(t)$ behaves as follows: for $t<t_{1}, \quad a(t) \propto t^{\frac{1}{2}}$ and for $t>t_{1}, \quad a(t) \propto t^{\frac{2}{3}}$. Here, $t<t_{1}$ corresponds to radiation dominated era and $t>t_{1}$ corresponds to matter dominated era.

Here we consider the effect of accretion on the life time of the rotating primordial black holes. Due to accretion in the radiation dominated era, the mass of the $\mathrm{PBH}$ increases and the accretion rate (which is taken to be proportional to the product of the surface area of the $\mathrm{PBH}$ and the energy density of the radiation [23]), is given by,

$$
\dot{M}_{a c c}=4 \pi f R_{B H}^{2} \rho_{R}
$$

where $\rho_{R}$ is the radiation energy density of the surrounding of the black hole. $r_{B H}$ is the radius of the outer horizon of the rotating black hole with mass $M$ and is given by, $R_{B H}=r_{+}=M+\sqrt{M^{2}-a^{2}}$ with $a(=J / M)$ being the rotation parameter and $J$ is the angular momentum. The rotating black hole solution satisfies the inequality $M^{2} \geq a^{2}$ in order to avoid a naked singularity. $f$ is the accretion efficiency. The precise value of $f$ is not known. The accretion efficiency could in principle depend on complex physical processes such as the mean free paths of the particles comprising the radiation surrounding the PBHs. Emission rates for massless particles from a rotating black hole and the subsequent evolution of the rotating black hole has been discussed in the nice work of Page [24] quite some time back. $\rho_{R}$ can be calculated from the Einstein's equation and is given by, $\rho_{R}=\frac{3}{32 \pi t^{2}}$ (we have taken $G=1$ ). Using these values, we get,

$$
\dot{M}_{a c c}=\frac{3 f}{8 t^{2}}\left(M+\sqrt{M^{2}-a^{2}}\right)^{2}
$$

This expression can be integrated to obtain the value of $M_{a c c}$ and it reduces to the nonrotating case in the limit $a=0$. One can notice from here that when $a^{2}$ is comparable to that of $M^{2}$, the rate of change of mass reduces to one fourth of the corresponding value obtained in the nonrotating case. In order to understand the exact effect of angular momentum parameter on the rate of accretion in the radiation 
dominated era, we have numerically plotted the variation of mass with change in the angular momentum parameter $a$ for a particular $\mathrm{PBH}$ formed at $t=10^{-22} \mathrm{sec}$ having accretion efficiency 0.5 in Figure 1. In our analysis, we assume that the initial mass $\left(M_{i}\right)$ of PBHs to be of the same as that of the horizon mass [25, 26]. One can see from Figure-1 that the rate of accretion decreases with increase in angular momentum parameter.

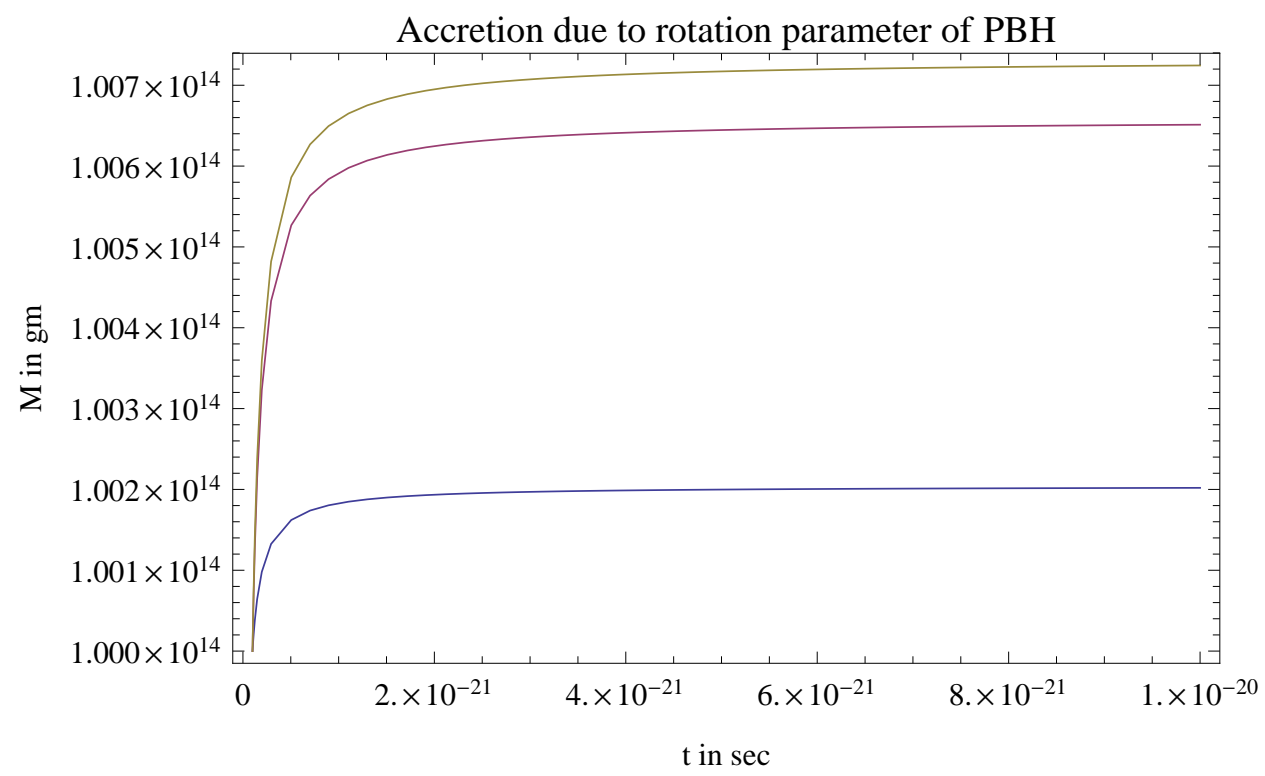

Figure 1: Variation of PBH mass for $a=M_{i}($ lower $), \frac{M_{i}}{2}($ middle $), \frac{M_{i}}{4}($ upper $) ; f=0.5$

Again by plotting the variation of mass of a particular rotating $\mathrm{PBH}$ formed at $t=10^{-22} \mathrm{sec}$ with an angular momentum parameter $a=\frac{M_{i}}{2}$ for different accretion efficiencies $f$ in Figure-2, we find that the mass of the PBH increases with increase in $f$ value as in the nonrotating case.

It is also worth noting that the upper bound of accretion efficiency $f$ is not fixed, but rather it varies with the angular momentum parameter $a$. For $a^{2}$ approaching $M^{2}$, the upper bound for $f$ is $\frac{8}{3}$ and for $a=0$, it reduces to the standard limit, i.e. $f<\frac{2}{3}$. In the case of nonrotating black hole in standard cosmology, it has been shown that the accretion can be effective in increasing the mass of the black hole and thereby increasing the life time of the primordial black hole.

It is worth mentioning here that in the hydrodynamic picture of the formation of the PBH during expansion of the early Universe [1, 27, 28], it has been shown through numerical calculations that the pressure gradient plays an important role in impeding the formation of PBHs. The rate of accretion of PBHs can reduce drastically by the pressure gradient. In case of the relativistic equation of state, initial perturbations have to be large enough in order to allow for the formation of PBHs. In the present context, 


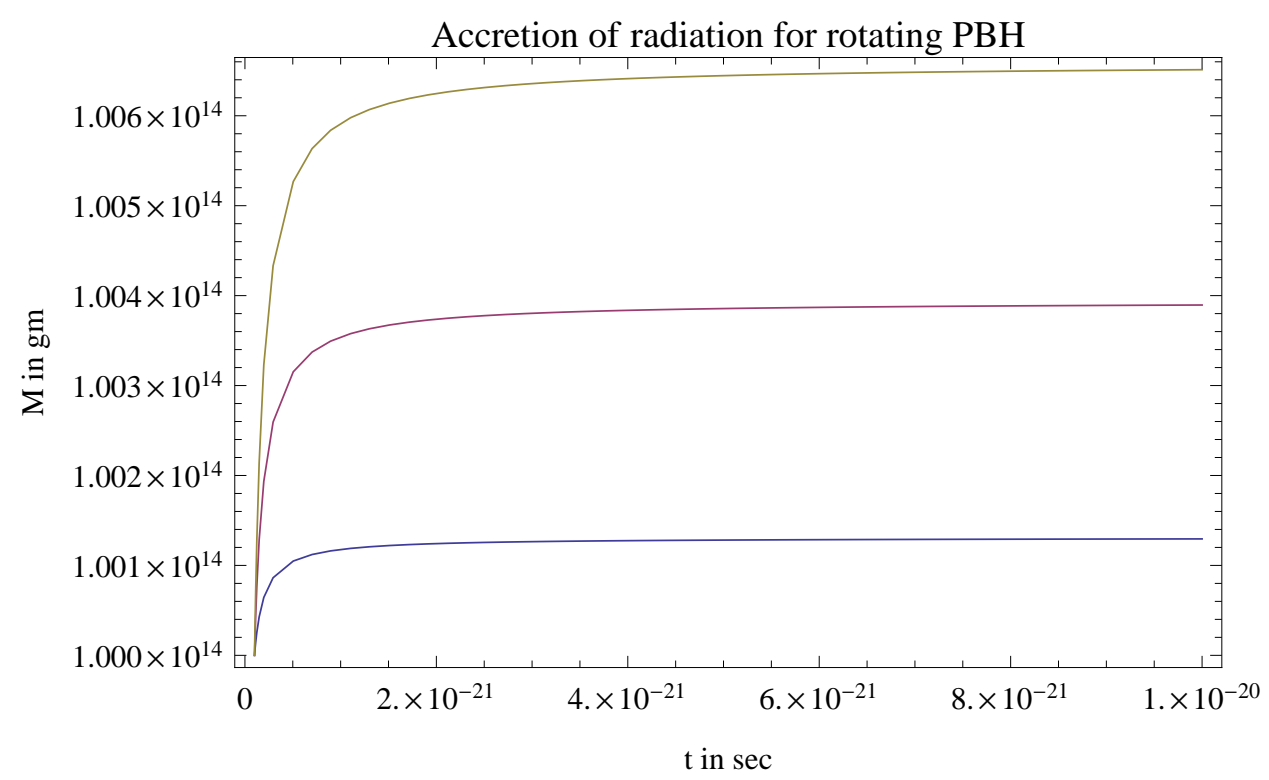

Figure 2: Variation of PBH mass for $f=0.1$ (lower), 0.3(middle), 0.5(upper), $a=\frac{M_{i}}{2}$

we have not considered the effect of pressure gradient on the accretion efficiency. Such consideration will need a full numerical computation which is beyond the scope of our paper.

\section{Evaporation of rotating $\mathrm{PBHs}$}

Now let us consider the evaporation of the rotating PBHs due to Hawking radiation. The rate of change of mass due to evaporation is given by,

$$
\dot{M}_{\text {evap }}=-4 \pi R_{B H}^{2} \sigma_{H} T_{B H}^{4}
$$

where, $\sigma_{H}$ is Stefan's constant multiplied by the number of degrees of freedom of radiation and $T_{B H}$ is the Hawking temperature for the rotating, uncharged black hole, given by,

$$
T_{B H}=\frac{\sqrt{M^{2}-a^{2}}}{4 \pi M\left(M+\sqrt{M^{2}-a^{2}}\right)}
$$

Using the expression for $T_{B H}$ and the radius of the outer horizon, one gets,

$$
\dot{M}_{\text {evap }}=-\left(\frac{\sigma_{H}}{64 \pi^{3}}\right) \frac{\left(M^{2}-a^{2}\right)^{2}}{M^{4}\left(M+\sqrt{M^{2}-a^{2}}\right)^{2}}
$$

Here one can see that when $a^{2}$ becomes comparable to that of $M^{2}$, the rate of change of mass during evaporation becomes negligibly small. So the rate of evaporation 
decreases with increase in angular momentum parameter. In principle, one should also consider the rate of change of angular momentum of the black hole due to the emission of the particles together with the rate of change of mass. However, for simplicity of the problem, we only look at the rate of change of mass due to evaporation.

The total rate of change of mass including both accretion and evaporation for the rotating $\mathrm{PBH}$ is given by,

$$
\dot{M}=\frac{3 f}{8 t^{2}}\left(M+\sqrt{M^{2}-a^{2}}\right)^{2}-\left(\frac{\sigma_{H}}{64 \pi^{3}}\right) \frac{\left(M^{2}-a^{2}\right)^{2}}{M^{4}\left(M+\sqrt{M^{2}-a^{2}}\right)^{2}}
$$

One can seee from the above equation that during the early period of evolution, the accretion term becomes dominant and at later time, evaporation dominates. One may assume that accretion takes place upto a time $t=t_{c}$ where both the accretion and evaporation rates are equal and then evaporation plays its role beyond $t_{c}$.

From the above equation (3.4), we obtain the expression for the time $t=t_{c}$ in terms of maximum mass $M_{c}$ and the accretion efficiency:

$$
t_{c}=\left(\frac{3 f}{32}\right)^{1 / 2} \alpha^{-1 / 2}\left(\frac{M_{c}^{2}\left(M_{c}+\sqrt{M_{c}^{2}-a^{2}}\right)^{2}}{M_{c}^{2}-a^{2}}\right)
$$

where, $M_{c}$ is the mass obtained from the accretion equation, i.e. $M_{c}=M_{\max }$.

Generally, PBHs are formed in the radiation dominated era and during their evolution in the radiation dominated era, they obey the evolution equation given in (3.4). However, in the matter dominated era, due to less dense surroundings, there is no appreciable absorption of matter-energy by the PBHs. So, in the matter dominated era, only the second term on the r.h.s. of (3.4) contributes.

For making the analysis better, we construct Table- 1 and 2 for showing the variation of evaporation time with respect to the angular momentum parameter and accretion efficiency respectively.

We may note from Table-I that the life time of $\mathrm{PBH}$ increases with increase in the angular momentum parameter, which happens due to more rapid decrease in evaporation rate than accretion rate with increase in angular momentum parameter. However, for small values of $a^{2}$, the evaporation time does not change significantly.

One can see from Table- 2 that the life time for the rotating PBH becomes longer by including the effect of accretion of radiation. One can check that in the limit $a=0$, the above expression for $t_{c}$ reduces to that of the nonrotating $\mathrm{PBH}$ i.e. $t_{c}=\sqrt{\frac{3 f}{2}} \alpha^{-1 / 2} M_{c}^{2}$.

We would like to mention here that in earlier work [24, 29, 30], it has been shown that angular momentum itself decreases with time nearly in the same order as the mass of the $\mathrm{PBH}$. This decrease in angular momentum also depends on the emission 


\begin{tabular}{|c|c|}
\hline \multicolumn{2}{|c|}{$t_{i}=10^{-22} s ; M_{i}=10^{14} g ; f=0.5$} \\
\hline$a^{2}$ & $t_{\text {evap }}$ \\
\hline 0 & $\sim 10^{13} \mathrm{~s}$ \\
\hline $10^{-9} M_{i}^{2}$ & $\sim 10^{13} \mathrm{~s}$ \\
\hline $10^{-7} M_{i}^{2}$ & $\sim 10^{18} \mathrm{~s}$ \\
\hline $10^{-5} M_{i}^{2}$ & $\sim 10^{22} \mathrm{~s}$ \\
\hline $10^{-3} M_{i}^{2}$ & $\sim 10^{24} \mathrm{~s}$ \\
\hline $10^{-1} M_{i}^{2}$ & $\sim 10^{28} \mathrm{~s}$ \\
\hline
\end{tabular}

Table 1: A rough estimate of evaporation time with change in angular momentum parameter

\begin{tabular}{|c|c|}
\hline \multicolumn{2}{|c|}{$t_{i}=10^{-23}$ sec $; M_{i}=10^{15} g ; a^{2}=10^{19}$} \\
\hline$f$ & $t_{\text {evap }}$ \\
\hline 0 & $3.333 \times 10^{13} \mathrm{~s}$ \\
\hline 0.2 & $3.363 \times 10^{13} \mathrm{~s}$ \\
\hline 0.4 & $3.394 \times 10^{13} \mathrm{~s}$ \\
\hline 0.5 & $3.409 \times 10^{13} \mathrm{~s}$ \\
\hline 0.6 & $3.425 \times 10^{13} \mathrm{~s}$ \\
\hline
\end{tabular}

Table 2: An estimate of evaporation time with change in accretion efficiency $\mathrm{f}$ with fixed angular momentum parameter

of particle species. So both accretion as well as evaporation of a PBH may be affected by this variation. Here we have not included the variation of angular momentum with time and the issues related to emission of massless or nearly massless particles.

\section{Summary and discussion}

In this work, we have considered the effect of accretion of radiation on rotating PBHs in homogeneous and isotropic FRW Universe. We find that the increase in angular momentum parameter decreases both the accretion and evaporation rate, but the rate of evaporation decreases more rapidly than the rate of accretion. This shows that rotation increases the life time of PBHs. It is also noted that the mass of the PBH increases with accretion efficiency as in the nonrotating case. Since Hawking radiation is supposed to carry away the angular momentum, it is worthwhile to have a detailed analysis of the evolution of the rotating PBHs taking into account the rate of change of angular momentum in the context of emission of massless or nearly massless particles with different spins. Here, we have not considered the effect of back reaction of the 
PBH evaporation [31] which is supposed to modify the radius of the horizon and the Hawking temperature of the black hole [32]. It is expected that such effects might affect

the evolution of PBHs. It is worth investigating these issues further in the context of $\mathrm{PBHs}$ with and without rotation.

\section{Acknowledgement}

We would like to thank L.P.Singh for useful discussions. 


\section{References}

[1] Ya. B. Zel'dovich and I. Novikov, Sov. Astron. 10, (1967) 602

[2] B. J. Carr, Astrophys. J. 201, (1975) 1

[3] M. Y. Kholpov, B. A. Malomed and Ya. B. Zeldovich, Mon. Not. R. Astron. Soc. 215, (1985) 575

[4] B. J. Carr, J. Gilbert and J. Lidsey, Phys. Rev. D 50 (1994) 4853

[5] M. Y. Kholpov and A. Polnarev, Phys. Lett. 97B, (1980) 383

[6] H. Kodma, M. Sasaki and K. Sato, Prog. Theor. Phys. 68, (1982) 1079

[7] D. La and P. J. Steinhardt, Phys. Rev. Lett 62, (1989) 376

[8] A. Polnarev and R. Zemboricz, Phys. Rev. D 43, (1988) 1106

[9] S. W. Hawking, Commun. Math. Phys. 43, (1975) 199

[10] K. J. Mack, J. P. Ostriker and M. Ricotti, Astrophys. J. 665, (2007) 1277

[11] D. Blais, C. Kiefer, D. Polarski, Phys. Lett. B 535, (2002) 11; D. Blais, T. Bringmann, C. Kiefer, D. Polarski, Phys. Rev. D 67, (2003) 024024; A. Barrau, D. Blais, G. Boudoul, D. Polarski, Annalen Phys. 13, (2004) 114

[12] B. J. Carr and S. W. Hawking, Mon. Not. R. Astron. Soc. 168, (1974) 399

[13] B. J. Carr, Astrophys. J. 206 (1976) 8

[14] J. D. Barrow and B. J. Carr, Phys. Rev. D 54, (1996) 3920

[15] B. J. Carr, K. Kohri, Y. Sendouda and J. Yokoyama, arXiv:0912.5297 [astro-ph.CO]

[16] A. S. Majumdar, Phys. Rev. Lett. 90, (2003) 031303

[17] R. Guedens, D. Clancy and A. R. Liddle, Phys. Rev. D66, (2002) 083509

[18] A. S. Majumdar and N. Mukherjee, Int. J. Mod. Phys. D14, (2005) 10955; A. S. Majumdar, D. Gangopadhyay and L. P. Singh, Mon. Not. R. Astron. Soc.385, (2008) 1467; B. Nayak, L. P. Singh and A. S. Majumdar, Phys. Rev. D80, (2009) 023529

[19] D. Dwivedee, B. Nayak, L. P. Singh, Int. J. Mod.Phys. D22, (2013) 1350022 
[20] B. Nayak and L. P. Singh, Pramana 76, (2011) 173

[21] B. Nayak, L.P. Singh, Eur.Phys. J. C71, (2011) 1837

[22] B. Nayak, M. Jamil, Phys.Lett. B709 (2012) 118-122.

[23] B. J. Carr, Astron. Astrophys. Trans. 5, (1994) 43

[24] D. N. Page, Phys. Rev. D14 (1976) 3260

[25] B. J. Carr, Phase transitions in the early universe: Theory and observations (Springer, 2000) 451-469

[26] B. J. Carr, Lect. Notes Phys. 631, (2003) 301

[27] D. K. Nadezhin, I. D. Novikov and A. G. Polnarev, Sov. Astron. 22(2) (1978) 129

[28] I. D. Novikov and A. G. Polnarev, Sov. Astron. 24(2) (1980) 147

[29] B. Carter, Phys. Rev. Lett. 33 (1974) 558

[30] D. N. Page, Phys. Rev. D13 (1976) 198

[31] C. O. Lousto and N. Sanchez, Phys. Lett. B212, (1988) 411

[32] L.Susskind and L.Thorlacius, Nucl. Phys. B382, (1992) 123 